\title{
A FRAMEWORK FOR NEW GENERATION TRANSPORTATION SIMULATION
}

\author{
Daiheng Ni \\ Department of Civil and Environmental Engineering \\ University of Massachusetts \\ Amherst, MA 01003
}

\begin{abstract}
This paper discussed the evolution and future trend of simulation in general domain and in transportation. Some challenges facing transportation modeling and simulation were identified. As an effort to address these challenges, a framework of new generation transportation simulation was developed. The framework is envisioned to be multiscale in resolution, parallel in execution, and driven by objects. The paper further discussed strategies of transportation simulation at a nanoscopic level which offers a level of modeling detail beyond the state-of-the-art.
\end{abstract}

\section{INTRODUCTION}

Both simulation in general and in transportation have evolved about half a century. Transportation simulation shares something in common with general simulation and, meanwhile, exhibits something particular in nature. Simulation is the process of generating an electronic version of the real world for many purposes including: (1) a tool for learning and understanding the physical world and its phenomena, (2) a basis on which it is risk-free to experiment and test assumptions, (3) a means to predict by allowing preview of possible outcomes, (4) a decision-tool to show the effects by means of visualization.

Transportation simulation is, meanwhile, a special application of simulation. For example, a traffic system is often thought as a queuing system, but it differs from a regular one such as a server. This is because (1) faster vehicles can overtake slower vehicles without having to wait behind, (2) vehicles can change lanes easily and this is in contrast to servers and guided vehicles such as trains, (3) capacity is not a point constraint in a process, but a continuous constraint over space/roadway, (4) congestion can occur unexpectedly in a traffic system, though bottlenecks are mostly observed at locations with reduced capacity, (5) traffic demands typically indicate strong time-series patterns rather than simply random distributions, (6) the human-in-the-loop nature renders a traffic system more fuzzy and intelligent than merely random.
Because of the above special nature of a traffic system, tools for transportation simulation are often designed specifically for such a purpose with less domain ambiguity. However, ambiguity often arises when different people talking about transportation modeling and simulation (TM\&S). Typically, for people doing research, TM\&S probably means using mathematic models to abstract part of the world and mimic its behavior in general; for information technology (IT) people, TM\&S frequently means implementing the mathematic model and developing corresponding software products; for transportation professionals such as people in government agencies and consulting firms, very likely TM\&S means using computerized software tools (simulators) to replicate and study the performance of a specific transportation system.

This paper falls into the first category, i.e. the paper focuses on general discussion on modeling philosophy in transportation simulation. In section two, a historical perspective is presented briefing the evolution of simulation in transportation. Challenges faced by transportation simulation are identified in section three, and modeling strategies in response to these challenges are outlined, based on which a framework of a new generation transportation simulation is developed. Section four focuses on a specific aspect of the framework - extremely detailed transportation simulation at a nanoscopic level. Modeling philosophy at this level of detail is conceptually presented including autonomous intelligent driver model, dynamic interactive vehicle model, and driver-vehicle-environment closed-loop system. Section five summarizes the paper.

\section{A HISTORICAL PERSPECTIVE}

The past decades have witnessed the evolution of simulation in general domain from time-driven modeling to eventdriven modeling to process-driven modeling, and the trend continues with object-driven modeling, data-driven modeling, and continuous modeling (Pegden 2005). A time-driven simulation advances simulation at fixed time steps. At each step, components of the simulation system is checked oneby-one and their states are updated accordingly. This mod- 
eling philosophy is straightforward and simple to implement and thus is extensively applied in early stage models. In some cases, system states may remain constant if time gaps between events are large and, as a result, computation power is wasted traversing these gaps. Under this circumstance, a more efficient approach may be event-driven modeling which updates system states at the occurrences of events. A process-driven simulation follows pre-defined logics and executes simulation steps sequentially, either in time domain or other domains or their combinations. A process-driven simulation requires that a modeler know the logical sequence of the simulation in advance and the simulation completes once the sequence has been traversed. In contrast, an object-driven simulation does not have a clearly defined modeling sequence. Rather, the simulation depends on interactions of objects which are entities in the system. Similar to object-oriented programming, an object possesses properties and behaves by means of methods. An object can be derived from some other objects inheriting their properties and methods or overriding them. A special type of object-driven simulation is an agent-driven simulation which typically applies to a system with human intelligence. An agent (or autonomous agent as frequently referenced in literature) is driven by goals and is able to achieve its goals by changing its behavior and adapting to its environment. All the above simulation scenarios are model-driven simulations in that the model controls the progress and all modeling activities occur within a single simulation process which typically captures modeling details at the same level. A recent emerging modeling philosophy is the so-called data-driven simulation. A data-driven simulation may be distributed and may be able to capture different levels of modeling detail. In essence, the progress of a data-driven simulation is controlled by input data. Different data may trigger different component models, different level of detail, and different simulation scopes, all of which can co-exist in a single simulation framework. All simulation efforts so far have been limited in discrete form because computers essentially work in discrete manner. Our eternal goal is to approximate real world systems which are continuous in domains such as time and space. In continuous modeling, system states change continuously over time and space with feedback from system entities and/or prior system states.

Echoing part of the above trend in a loose sense, simulation in transportation has evolved from macroscopic simulation to mesoscopic simulation to microscopic simulation, and the trend continues. A macroscopic simulation (Michalopoulos 1984; Michalopoulos and Lin 1986; Newell 1993a; Newell 1993b; Newell 1993c; Daganzo 1994; Daganzo 1995a; Daganzo 1995b; Ni et al. 2005; Ni and Leonard 2005) is purely a time-driven process in that, at each time step, traffic as a compressible fluid is pumped into the simulation system, moves forward link-by-link, and exits the system eventually. Central to the flow of traffic through the system is the conservation law which states that traffic entering a section of road should be equal to traffic exited plus any storage (Lighthill and Whitham 1955; Richards 1956). A taxonomy of macroscopic models can be found in ( $\mathrm{Ni}$ et al. 2005). Rather than treating traffic as a continuous fluid, a mesoscopic simulation (Van Aerde and Yager 1988; Morrison and Loose 1995; Smith et al. 1995; Van Aerde 1995; LANL 1999) models traffic as discrete particles without mass and personality. Much like a process-driven simulation, these particles traverse a discretized time-space grid, hopping from one cell to another governed by some pre-defined local rules such as maximum speed constraints. Though still time-driven in nature, a microscopic simulation adds some flavor of object-driven simulation. Driver-vehicle units in a microscopic simulation are similar to objects in that they possess properties (e.g. perception-reaction times, aggressiveness, preferences, etc.), behave by means of methods (carfollowing logics, lane-changing logics, and gap-acceptance logics), and are driven by goals (starting from their origins and arriving at their destinations). Microscopic simulation represents the current state-of-the-art in transportation simulation and a taxonomy of microscopic models is available from the Next Generation Simulation (NGSIM) supported by Federal Highway Administration. Seemingly simple to model, the complexity of transportation simulation comes from the huge number of vehicles involved and the difficulty to keep track of their states and interactions, needless to say other system components such as roadways and signals. After decades of continuous effort, transportation simulation has evolved to the current state featuring models with varying levels of detail and over a hundred simulators, some of which have been widely applied in various aspects of transportation engineering.

\section{NEW GENERATION SIMULATION FRAMEWORK}

\subsection{Challenges of transportation simulation}

With computers become more and more powerful, there is a trend to analyze and address transportation problems from a system perspective involving increasingly large sizes and fine details. This brings about a number of challenges to the state-of-the-art transportation simulation:

(1) Lack of details. Though microscopic simulation has sufficed our past and some of today's needs, its limitation in terms of modeling details has been more and more widely recognized. As a matter of fact, many traffic engineers feel difficulty in mimicking field traffic patterns using current limited logics on driver behaviors and vehicle responses. The main issue is that vehicles in the virtual environment are not driven the same way as they are in the real world. For example, vehicle acceleration and deceleration performances have been unrealistic because of the use 
of massless vehicle models. On the other hand, deterministic mathematical approach has demonstrated only limited success in mimicking driver behavior where artificial intelligence approach including fuzzy logics and neural networks may be more appropriate. Though longitudinal vehicle movement accounts for major aspects of traffic operation, inappropriate lateral movements are frequently found to be the causes of some operational problems, especially those of high impacts such as crashes and rollovers. As a matter of fact, NGSIM is actively seeking solutions to add more details in TM\&S in terms of behavior models.

(2) Lack of flexibility. Traditional simulation approach treats everywhere in a transportation system with the same level of detail. This poses challenges to some applications having competing requirements on both scale and detail. For example, modeling a regional transportation network inevitably sacrifices local details such as intersection operation, while achieving local details limits the network size that an application can handle.

(3) Costly to build models. Building models involves preparing data for the subject network and coding the network into a simulation model. As the size of the network becomes large, the double complexity of network structure and data preparation can grow exponentially. Needless to say the resources needed to calibrate and validate the model. As a result, simulating a complex transportation system sometimes can be prohibitive due primarily to the cost of building the model.

\subsection{The framework of new generation simulation}

In response to the challenges identified above, a new generation transportation simulation is envisioned to be multiscale in resolution, parallel in execution, and driven by objects.

\subsubsection{Multi-scale resolution}

A simulation may not necessarily need the same resolution everywhere in the subject transportation network. For example, management of a regional transportation network concerns more on its overall throughput, in which case a macroscopic may be more appropriate to achieve modeling scale. As congestion built up at an intersection/interchange, the traffic analyst may be interested in identifying what factors contribute to the congestion and how it evolves. This scenario necessitates simulation at microscopic level or even more detailed level such as nanoscopic simulation. For some part of the network, the analyst may want to learn more details about traffic dynamics while still overseeing the traffic flow, an intermediate scale such as mesoscopic simulation may be more appropriate.

Figure 1 illustrate such a scenario with multi-scale resolution. Simulation of Atlanta regional transportation network is performed at a macroscopic level (the background map) to achieve modeling scale and maintain efficiency. Two interstates I-75 and I-85 merge and diverge at Brookwood interchange where traffic analysts want to obtain more details here, so a closer view is obtained by "zooming in" to a mesoscopic level (the top local view). Downtown connector, the confluence of I-75/I-85, is frequently the root of congestion. An even closer view at a microscopic level (the middle local view) may reveal the instability of traffic and possible cause of traffic breakdown. A view with higher resolution such as nanoscopic level (the bottom view) is always helpful to examine the interactions between vehicles and identify means to improve traffic flow based on control at individual vehicle level. Notice that, rather than applying the same modeling resolution everywhere in the network, different levels of detail are applied at different parts of the network and they co-exist in the same simulation. This modeling technique is expected to improve simulation flexibility and address simultaneous requirements of scale and detail.

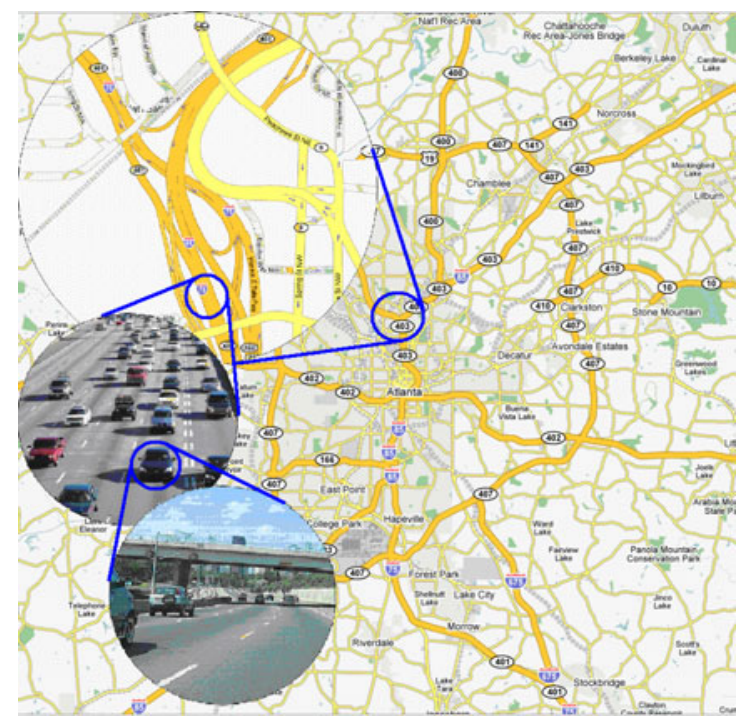

Figure 1: Multi-Scale Resolution Simulation

\subsubsection{Parallel execution}

In line with the need to satisfy both scale and detail, a simulation does not have to run in a monolithic fashion. Multiple simulation processes can communicate and synchronize such that each simulation takes care of part of a larger transportation network. This technique can be implemented in a single computer executing multiple simulation processes in parallel or distribute the processes to multiple computers/locations to share workload and information.

Closely related to the parallel/distributed simulation is its convenience to incorporate data-driven applications where locally distributed simulation processes are easier to 
take care of changes in traffic pattern such as sudden surges, accidents, and emergencies. These pattern changes then steer the simulation processes to enter next level of detail as appropriate.

There are generally two approaches to parallelism: functional parallelism and spatial parallelism. Both of them are desirable for the application of interest. For example, the functional parallelism allows one or more lowlevel, local microscopic simulation to co-exist with a highlevel, global macroscopic simulation; the spatial parallelism is ideal to partition a large network into several small but more detailed sub-networks spatially and map them to different processors.

\subsubsection{Object models}

Actually, the object-oriented paradigm was originated in simulation world and was adopted in programming world (Pegden 2005). One of the advantages of thinking in objects is to reuse codes and save time in repeated works, which opens the door of simplifying model building. In transportation simulation, an object can be anything ranging from links, nodes, signs, signals, vehicles, drivers, and even the model itself. Therefore, it is important to coin the concept of object model, i.e. a model can be an object and an object can be a model. An object can have properties, carry goals, and behave by methods. For example, a signal has timing plans and behaves by indicating right of ways. A vehicle has dimensions, mass, and power and behaves by carrying out dynamic response to control strategies. A driver has personalities and preferences and behaves by determining vehicle control strategies. Importantly, the driver's behavior is motivated by its goal - traveling from point A to point B subject to certain constraints such as minimizing travel time. What make objects attractive are their ability to be encapsulated with data, instantiated to spawn multiple copies, and modified to generate new objects based on their parents to exhibit new properties, methods, and goals. Driven by their goals, objects can work autonomously and adapt their behavior to new settings. This is much the same way that real word systems work - everybody acts on its own schedule, yet there is a universal time to coordinate the actions of all of those involved. This modeling philosophy partly echoes the parallel execution discussed above.

\section{NANOSCOPIC TRANSPORTATION SIMULATION}

Though real world systems can be examined with continuously finer resolution, transportation simulation has to be "zoomed in" by discrete levels, i.e. macroscopic, mesoscopic, microscopic, and intuitively nanoscopic. Our current knowledge has covered the first three levels. In this section, we try to extend our understanding to the last level in an exploratory and conceptual manner.

To illustrate the difference in modeling philosophies of these levels and facilitate subsequent discussion, we make the following analogy. Suppose one is observing traffic $10,000 \mathrm{~m}$ above the ground, traffic behaves as a compressible fluid whose states (speed, flow, and density, etc.) propagate back and forth like waves. This is a scenario of macroscopic simulation. If one lowers to $3,000 \mathrm{~m}$, the sense of waves recedes and a scene of particles emerges. A vehicle behaves as a particle hopping from one cell to another governed by some predetermined logics. This is a scenario of mesoscopic simulation. If one lowers even more to $1,000 \mathrm{~m}$, the scene is dominated by moving particles which interact with each other so as to maintain safe positions in the traffic stream. This is a scenario of microscopic simulation as well as the state-of-the-art. Nanoscopic simulation is fundamentally different from the above modeling philosophies. Continuing with the above analogy, nanoscopic simulation provides a perspective as though one were to get down to the ground and drive in the traffic stream. What one sees now is neither wave nor particle, but a complicated nanoscopic system consisting of drivers, vehicles, and environment (e.g. roadway, signs, signals, etc.). Drivers collect information and make control decisions in terms of steering, acceleration, and deceleration. Vehicles dynamically respond to drivers by executing the control decisions and moving on the ground. Feedback from vehicle dynamics, together with information from the environment, constitutes the basis for drivers to make control decisions in the next step and the above process goes on and on. Traffic operation is simply the movement and interaction of all vehicles in the system over time and space. This is a scenario of nanoscopic simulation.

\subsection{Autonomous intelligent driver model}

A driver is a special object in simulation, commonly referred to as an autonomous agent. It is autonomous because it acts on its own, i.e. it is driven by goals and is able to adapt to the changing environment. It is intelligent because it is able to reason, i.e. using the current context as a key to find solutions from its knowledge base and past experience.

Thinking in an object-oriented (O-O) paradigm, a driver object has properties, behaves by methods, and pursues goals. More specifically, driver properties include aggressiveness, alertness, perception-reaction time, decision thresholds, preferences (lane and speed), etc. Driver goals can be expressed as a combination of the following constraints: origin and destination, safety and security, travel time, and other costs. Driver methods involve a complicated reasoning process to determine driving control strategies including acceleration, deceleration, and steering based on current context. 
Figure 2 illustrates the reasoning process of the Autonomous Intelligent Driver model which consists of three parts: inputs, the driver model, and outputs. Driver inputs come from environment as well as vehicle feedback.

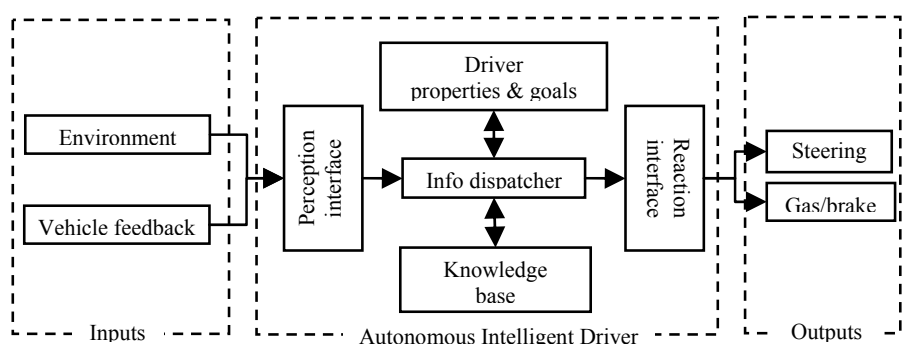

Figure 2: Autonomous Intelligent Driver Model

The environment here generally refers to the entire system including drivers, vehicles, pedestrians, roadway infrastructure, traffic control devices, roadsides, abutting lands, nearby business, etc. From a driver's perspective, there are local environment and global environment. The local environment consists of everything in the vicinity around the driver such as roadway geometry, traffic control devices, other surrounding vehicles, distances to other vehicles, deviation from target lane, available driving spaces, etc. Local environment affects the driver's immediate driving tasks such as acceleration and deceleration. The global environment is actually the mapping of the entire system in the driver's brain such as alternative routes to destinations, traffic control devises along the routes, anticipated travel times on the routes, congestions and incidents elsewhere, points of interests, etc. Global environment affects the driver's long turn driving tasks such as route choice and diversion. Vehicle feedback includes dynamic responses generated from a vehicle and perceived by its driver, such as vehicle position, speed, acceleration, yaw rate, etc.

As an intelligent agent, a driver is able to (a) respond in a timely fashion to changes in the environment, (b) exercises control over his/her own actions, (c) pursue a goal by which to drive his/her actions, (d) communicate with other agents, and (e) change his/her behavior based on previous experience. These aspects of human intelligence can be modeled by artificial intelligence (AI) such as adaptive neuro-fuzzy inference system (ANFIS). In solving complex, non-linear, and dynamic problems such as vehicle control, past research has shown that AI appeared to be more efficient than conventional deterministic mathematic approach. In addition, AI is able to mimic the natural way of driving a vehicle. Based on the above considerations, the intelligent driver consists of the following components: (i) Perception interface which mimics the way that information enters the driver's eyes and forms an image in the driver's brain. Since the driver, as a human being, can only take information in fuzzy terms, crispy information from outside needs to be fuzzified before registered in the driver's brain. (ii) Reaction interface which functions as the reverse of the perception interface, i.e. fuzzy decisions made by the driver need to be defuzzified to crispy information before execution on vehicles. (iii) Driver properties and goals as explained before. (iv) Knowledge base which consists the experiences and decision rules that govern driving behavior. For example, when there is no obstacle in front and the vehicle is running slow, the knowledge base may suggest speeding up. (v) Information dispatcher which is actually the central processing unit of the driver model. Three sources of information are critical to a driving task: environment information, vehicle feedback, and driver properties. The dispatcher receives information from the three sources, queries the knowledge base, gets answers from it, determines control strategies, and sends them to decision queue for execution.

Outputs of the model are strategies of vehicle control such as steering wheel angle, gas, and brake, of which the latter two can be simplified as one. This is exactly how drivers control their vehicles in the real world.

Modeling drivers as objects provides great flexibility and simplification to model building. For example, driverrelated data are encapsulated in driver objects and only the objects can change their states; driver input patterns trigger object-specific actions; specialized objects (pedestrians, bicyclists, auto drivers, heavy vehicle drivers, etc.) can be derived from existing objects (generic drivers); new objects can be built by combining existing objects.

\subsection{Dynamic interactive vehicle model}

Thinking again in O-O paradigm, a vehicle object has properties, behave by methods, but no goals. Vehicle properties include mass, power, dimensions, etc. Vehicle methods can be expressed as a set of dynamic equations mapping driver control strategies to vehicle responses. A vehicle object is dynamic because its responses change dynamically as a function of driver control strategies and it captures vehicle dynamics including mass and acceleration (longitudinal and lateral). The object is also interactive because its responses depend on driver control strategies and, in return, influence driver decision-making.

Figure 3 illustrates how a vehicle object/model works. Three parts are involved: inputs, the vehicle model, and outputs.

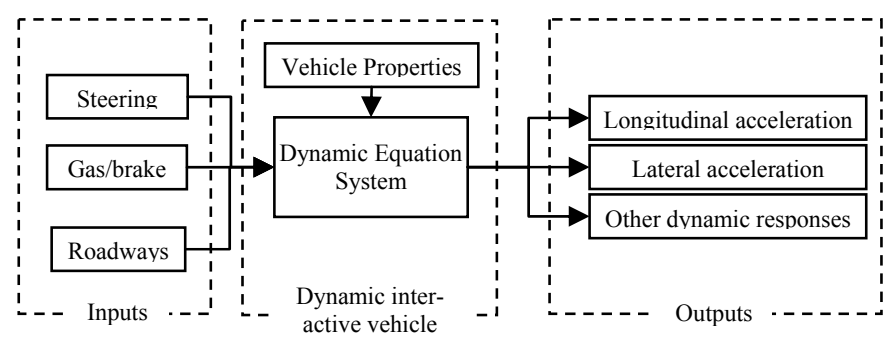

Figure 3: Dynamic Interactive Vehicle Model 
Inputs of the vehicle model come from two sources: the driver and the environment. Inputs from the driver include steering wheel angle, throttle position, and brake position. The last two can be combined as one since they both control acceleration (deceleration is negative acceleration). Inputs from environment is the physical space in which the vehicle operates, such as roadway surfaces, lanes, curves, and resistances.

The vehicle model consists of vehicle-specific information and vehicle-generic information. Vehicle-specific information contains vehicle properties such as mass, dimension, and engine power. Vehicle-generic information is actually a set of dynamic equations describing the dynamic performance of a class of vehicles, such as acceleration/deceleration and steering performance.

Outputs of the vehicle model are vehicle dynamic responses, of which the following are of particular interest: (i) longitudinal acceleration which describes vehicle longitudinal movement and affects the driver's choice of speed and car-following behavior; (ii) lateral acceleration which describes vehicle lateral movement and affects lane change, turning, overturn, etc. (iii) yaw rate which describes vehicle stability under steering and exerts some influence on the driver to decide appropriate combination of speed and turning. Yaw rate is also closely related to vehicle rollover.

\subsection{Driver-vehicle-environment closed-loop system}

The above discussion presents an autonomous intelligent driver model and a dynamic interactive vehicle model. Working together, the two models form a driver-vehicle closed-loop system which constitutes a basic building block of roadway traffic - a new object derived by combining the previous two. Many such objects as well as roadways, traffic control devices, and other objects related to traffic operation constitute a general environment in which the driver-vehicle objects operate. The interactions among drivers, vehicles, and environment are summarized in Figure 4 which illustrates the architecture of nanoscopic transportation modeling and simulation.

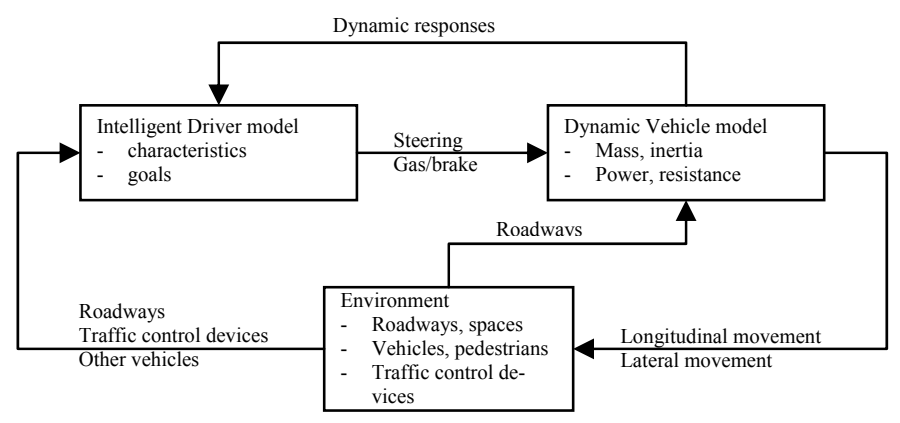

Figure 4: Driver-Vehicle-Environment System
In this architecture, the driver receives information from the environment such as roadways, traffic control devices, and the presence of other vehicles. The driver also receives information from his/her own vehicle such as speed, acceleration, and yaw rate. These sources of information, together with driver properties and goals, are used to determine driving strategies (such as steering and gas/brake). The driving strategies are fed forward to the vehicle which also receives roadway information from the environment. These sources of information, together with vehicle properties, determine the vehicle's dynamic responses based on vehicle dynamic equations. Moving longitudinally and laterally, the vehicle constitute part of the environment. Other vehicle dynamic responses such as speed, acceleration, and yaw rate are fed back to the driver for determining driving strategies in the next step. Therefore, the architecture creates an environment, in which each driver-vehicle object is an autonomous agent which is driven by goals and is able to achieve the goals by moving through the environment. Thus traffic operation is simply the movements and interactions of all vehicles in the environment.

It can be seen conceptually that the implementation of the above nanoscopic simulation will be a very difficult task and the execution of such a nanoscopic simulation will be heavyweight. These difficulties echo the need for the multi-scale resolution simulation framework, i.e. a lightweight, low-resolution simulation takes care of networkwide performance, while a heavyweight, high-resolution simulation reveals details in a sub-network or a point on roadway.

\section{SUMMARY}

This paper discussed some commonalities and differences between simulation in general domain and simulation in transportation, as well as their evolution of modeling philosophies. A few challenges are faced by the state-of-theart transportation simulation in an effort to meet today and tomorrow's needs in transportation modeling and simulation. These challenges include: (1) a lack of details in a sense that vehicles in the virtual environment are not driven the same way as in the real world, (2) a lack of flexibility in a sense that existing models have difficulty to meet the simultaneous requirements on scale and detail, and (3) costly to build models in a sense that data collection and model coding are sometimes too expensive to warrant a simulation application.

To deal with the above challenges, a framework of new generation transportation simulation was proposed. Future transportation simulation is envisioned to be multiscale in resolution, parallel in execution, and driven by objects. More specifically, future transportation simulation is expected to be able to accommodate different levels of detail in the same simulation. Macroscopic simulation facili- 
tates large scale modeling, microscopic simulation reveals more local details, while mesoscopic simulation balances scale and detail. Future transportation needs to run parallel and/or in a distributed manner. Such a consideration comes from the need of sharing workload and information as well as to facilitate data-driven applications. Future transportation simulation is envisioned to be driven by objects which are system components capable of acting on their own yet interacting with other objects. By means of encapsulation, instantiation, and overriding, object-driven simulation is expected to render a simulation more realistic and efficient.

Moving along the line of macroscopic, mesoscopic, and microscopic simulation, the next step to improve modeling detail is nanoscopic simulation. This can be achieved by incorporating an autonomous intelligent driver model, a dynamic interactive vehicle model, and integrate both in a driver-vehicle-environment closed-loop system. The driver model is capable of capturing environment information and vehicle feedback to control a vehicle in terms of accelerating, decelerating, and steering. The vehicle model executes the above control strategies and makes dynamic responses accordingly. The driver-vehicle object interacts with other similar objects as well as other system objects such as roadways and signals. Thus traffic operation is modeled as actions and interactions of all objects in a driver-vehicle-environment closed-loop system.

\section{REFERENCES}

Daganzo, C. F. (1994). The cell transmission model: A dynamic representation of highway traffic consistent with the hydrodynamic theory. Transportation Research B 28(4): pp. 269-287.

Daganzo, C. F. (1995a). The Cell Transmission Mode, Part II: Network Traffic. Transportation Research B 29(2): pp. 79-93.

Daganzo, C. F. (1995b). A Finite Difference Approximation of the Kinematic Wave Model of Traffic Flow. Transportation Research B 29(4): pp. 261-276.

LANL (1999) Transportation Analysis Simulation System (TRANSIMS). LA-UR-99-1658. Los Alamos National Laboratory.

Lighthill, M. and G. Whitham (1955). On kinematic waves II. A theory of traffic flow on long crowded roads. Proc. Royal Society of London, Part A 229(1178): pp. 317-345.

Michalopoulos, P. G. (1984). Dynamic freeway simulation program for personal computers. Transportation Research Record 971: pp. 68-79.

Michalopoulos, P. G. and J. Lin (1986). Integrated Modeling of Freeway Flow and Application to Microcomputers. Transportation Research Record 1091: pp. 2528.
Morrison, J. and V. Loose (1995) TRANSIMS Model Design Criteria As Derived From Federal Legislation. DOT-FTA-T-95-21. Los Alamos National Laboratory.

Newell, G. F. (1993a). A simplified theory on kinematic waves in highway traffic, Part I: general theory. Transportation Research B 27(4): pp. 281-287.

Newell, G. F. (1993b). A simplified theory on kinematic waves in highway traffic, Part II: queueing at freeway bottlenecks. Transportation Research B 27(4): pp. 289303.

Newell, G. F. (1993c). A simplified theory on kinematic waves in highway traffic, Part III: multi-destination flows. Transportation Research B 27(4): pp. 305-313.

$\mathrm{Ni}$, D., et al. (2005). The Network Kinematic Waves Model: A Simplified Approach to Network Traffic. Journal of Intelligent Transportation Systems: Technology, Planning, and Operations. In print.

Ni, D. and J. D. Leonard (2005). A Simplified Kinematic Wave Model at a Merge Bottleneck. Applied Mathematical Modelling, Elsevier Science: In Print.

Pegden, C. D. (2005). Future Directions in Simulation Modeling. Winter Simulation Conference 2005.

Richards, P. I. (1956). Shock waves on the highway. Operations Research 4: pp. 42-51.

Smith, L., et al. (1995). TRANSIMS: Transportation Analysis And Simulation System. Fifth National Conference on Transportation Planning Methods Applications-Volume II.

Van Aerde, M. (1995) INTEGRATION - Release 2. User's Guide. the Transportation Systems Research Group.

Van Aerde, M. and S. Yager (1988). Dynamic Integrated Freeway/Traffic Signal Networks: Problems and proposed Solutions. Transportation Research A 22(6): pp. 435-443.

\section{AUTHOR BIOGRAPHY}

DAIHENG NI is an Assistant Professor of Civil Engineering at University of Massachusetts Amherst. He was previously a post-doctoral fellow at School of Civil and Environmental Engineering at Georgia Institute of Technology. He holds the degrees of M.Sc. in Civil Engineering and M.Sc. in Industrial Engineering and Ph.D. in Civil Engineering from Georgia Institute of Technology. His research interests include traffic flow theory and simulation, intelligent transportation systems, traffic sensing and information technology, and transportation logistics and optimization. His email address is $<$ ni@ecs. umass. edu $>$ and his personal web page is <http://www.ecs.umass.edu/cee/ $\mathrm{ni} />$. 